\title{
IMPROVING ANALYTIC NETWORK PROCESS REPORTING
}

\begin{abstract}
A review of more than 100 ANP studies published in 2015 shows that the report of these studies is either deficient or incomplete, to the point that it casts a shadow on the validity of their conclusions. In this study we identify key elements that must be present to ensure the validity, replicability and overall quality of the reported ANP study.
\end{abstract}

Keywords: ANP reporting, ANP studies.

\section{Introduction}

Since its appearance in the MCDM scene, many studies have been done using the Analytic Network Process -ANP- (Saaty, 2005). We contend in this study that most ANP studies are reported in the literature in a way that does not allow one to check the overall validity of the model or even better to allow for the replication of the published study. Furthermore, this is particularly acute in the report of group decision-making studies.

This problem is greatly due to the lack of accepted standards for ANP reporting although some general guidelines, such as judgment aggregation, have been provided in the literature (Saaty and Peniwati, 2007). Through our review of a large number of recent ANP studies, we have been able to confirm this proposition as well as to identify different ways to alleviate this problem.

\section{Literature Review}

We reviewed the web of science database to search for ANP articles. This review was done in two stages: first, to check if any articles related to ANP reporting had been published during the past years and next, to review if the ANP studies published could be considered valid and susceptible of replication. We did not obtain any results in the first phase but we found 743 results. To make the results more manageable we selected only articles published in 2015 and obtained 105 ANP published articles. These were the articles that were painstakingly reviewed one by one.

\section{Hypotheses/Objectives}


The objectives of this study are twofold: first, to check if ANP study reporting is done with a minimum number of requirements as to assess their overall validity and eventual replication. Second, to recommend ways to improve ANP reporting which may lead eventually to the development of ANP reporting standards.

\section{Research Design/Methodology}

The criteria for the proposed ANP best practices were developed using an expert opinion approach for model development. The first two authors have significant experience using and teaching ANP as well as reviewers and one of them is a seasoned editor. From the dataset of ANP published articles, we identified if different ANP model elements such as criteria definitions, influence matrix, cluster comparison matrix, un-weighted and weighted supermatrices were present or absent in the report. Next, authors discussed and agreed to the minimum set of requirements to ensure the study validity, ability to be replicate and overall quality, defined as the degree of the excellence (Merriam-Webster, 2018) of the study.

\section{Data/Model Analysis}

Table 1 summarizes the variables, whose presence was deemed necessary for the proper validity/replication and overall quality assessment of the papers reviewed in this study.

Table 1 - Summary of ANP Report Requirements for Validity/Replication*

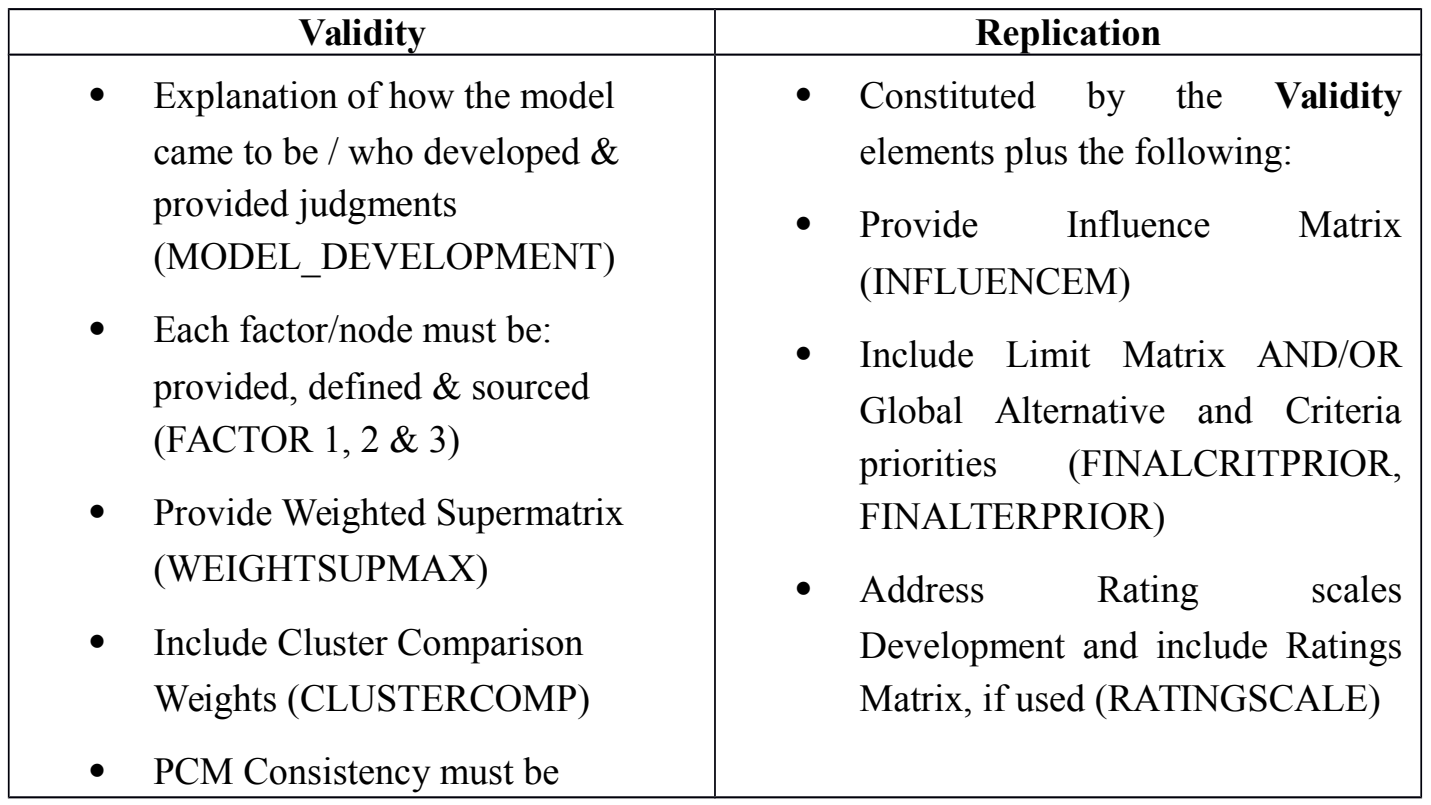




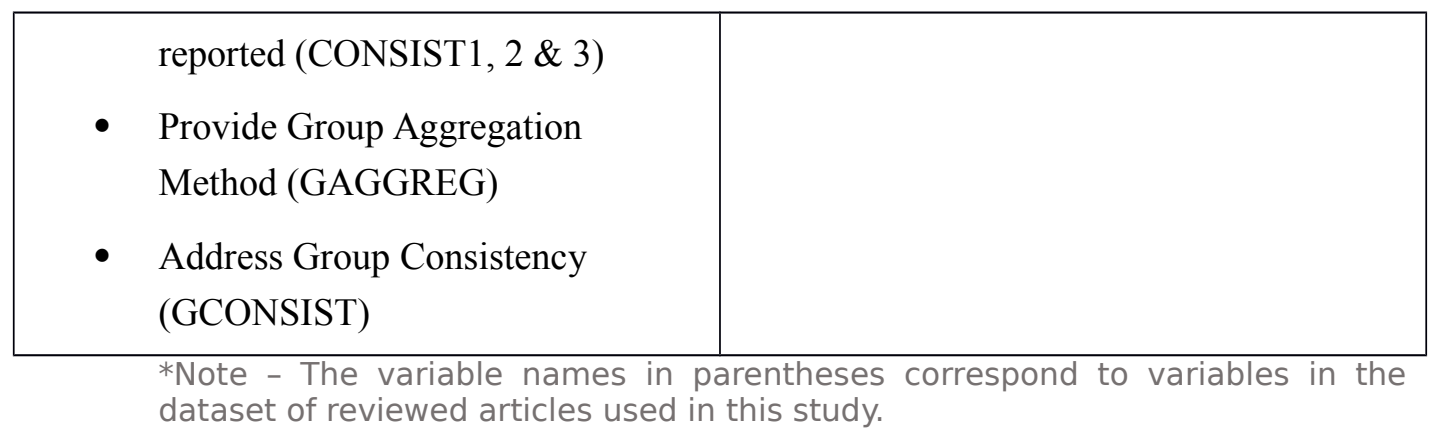

\section{Limitations}

Given that the above checklist of critical reporting factors was done solely by the authors, this checklist must be corroborated in practice by ANP scholars using the checklist for the purpose of either writing, reading or editing papers for journal publication.

\section{Conclusions}

In conclusion, our research -based on the detailed review of more than 100 ANP published studies- provides the following contributions to the ANP literature:

- Raises an alarm about the need to follow a minimum set of practices to ensure the ANP study validity, replication and overall quality of the study.

- Suggests key variables to check to address the above concerns in the hope that this approach will eventually lead to the development of accepted ANP reporting standards.

\section{Key References}

Merriam-Webster (2018). Merriam-Webster Dictionary Online. Retrieved from https://www.merriam-webster.com/dictionary/quality

Saaty, T,. L. (2005). Theory and Applications of the Analytic Network Process. RWS Publications: Pittsburgh, PA.

Saaty, T.L., \& Peniwati, K. (2007). Group decision-making: Drawing out and reconciling differences. Pittsburgh, PA: RWS Publications.

\section{Appendices}


ISAHP Article: A Style Guide for Paper Proposals To Be Submitted to the International Symposium on the Analytic Hierarchy Process 2018, Hong Kong, HK.

While not ready at this point we intend to provide the audience with a checklist form titled "Cheklist for improving your ANP report" 